\title{
Comparison of in vivo and in vitro digestion of polyphenol composition in lingonberries: potential impact on colonic health
}

\author{
E. M. Brown ${ }^{1}$, S. Nitecki ${ }^{1}$, G. Pereira-Caro ${ }^{2}$, G. McDougall ${ }^{3}$, D. Stewart $^{3}$, I. Rowland ${ }^{4}$, \\ A. Crozier ${ }^{2}$ and C. I. R. Gill ${ }^{1}$ \\ ${ }^{1}$ Northern Ireland Centre for Food and Health, University of Ulster, Coleraine, N. Ireland, BT52 1SA, UK, ${ }^{2}$ College of \\ Medical, Veterinary \& Life Sciences, Graham Kerr Building University of Glasgow G12 8QQ, ${ }^{3}$ Environmental \& \\ Biochemical Sciences Group, The James Hutton Institute, Invergowrie, Dundee, DD2 5DA, UK and ${ }^{4} H u g h$ Sinclair \\ Unit of Human Nutrition, Department of Food \& Nutritional Sciences, University of Reading, Whiteknights, Reading \\ RC6 6AP, UK
}

Evidence from epidemiological studies suggests that diets rich in fruit and vegetables may contribute to a reduced risk of colorectal cancer (CRC). A recent meta-analysis of risk factors associated with colorectal cancer reported a decreased risk associated with fruit consumption $(\mathrm{RR}=0.85,95 \% \mathrm{CI}=0.75-0.96$ for 3 servings/day) and also emphasized that low fruit and vegetable consumption was associated with a moderately increased risk of $\mathrm{CRC}^{(1)}$. Lingonberry (Vaccinium vitis-idaea $L$ ) is a popular edible berry in Scandinavian countries and is increasing in popularity across Europe. Lingonberry possesses a complex (poly)phenolic profile, which may contribute to its putative anticancer activity. Following consumption and digestion, limited uptake from the small intestine results in berry (poly)phenolic compounds entering the colon, where they are subject to microbiota-mediated metabolism ${ }^{(2)}$. Therefore, it is likely that the colonic epithelium is exposed to phenolic metabolites as well as the original parent compounds.

The aim of this study was to evaluate the impact of in vivo and in vitro digestion on phenolic composition and bioactivity of lingon berries. Lingonberry extracts obtained after simulated in vitro digestion (IVDL) and subsequent faecal fermentation (IVFL) were com-

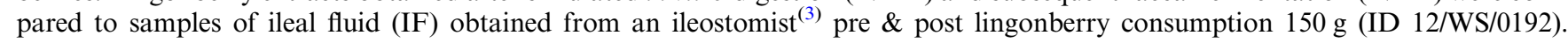
Bioactivity of the extracts was tested using a physiologically-relevant dose range $(0-50 \mu \mathrm{g} / \mathrm{ml}$ gallic acid equivalents) over a 24 hour exposure period using in vitro colonocyte models of colorectal carcinogenesis (Comet assay HT29, mutagenicity assay HT29 $\mathrm{G}^{17}$ neo, Matrigel invasion assay HT115) ${ }^{(4)}$. LC-MS analysis confirmed that in vitro digestion altered the (poly)phenol composition relative to the original lingonberry and similar patterns were observed for the ileal fluid. On the other hand, the IVFL sample had high levels of simple aromatic components. Digested and fermented extracts exhibited significant $(p<0 \cdot 05)$ anti-genotoxic, antimutagenic and anti-invasive effects compared to the appropriate controls (ANOVA, Post Hoc Dunnett T test) in all in vitro models. The ileal fluid demonstrated a significant reduction in DNA damage $(p<0 \cdot 05)$, but at a higher total polyphenol concentration $(\mu \mathrm{g} / \mathrm{ml}$ GAE $/ \mathrm{ml}$ ) than the IVDL. Despite extensive structural modification following digestion and fermentation, lingonberry extracts retained their bioactivity. These data reinforce the need for screening studies to consider the impact of digestion when investigating bioactivity of dietary phytochemicals.

1. Johnson CM, Wei C, Ensor JE, Smolenski DJ, Amos CL, Levin B, Berry DA (2013) Cancer Causes Control 24, 1207-1222.

2. Del Rio D, Borges G, Crozier A (2010) Br J Nutr, 104, S67-90.

3. Gonzalez-Barrio R, Borges G, Mullen W, Crozier A. (2010). Agric. Food Chem., 58, 3933-3939.

4. Brown EM, McDougall GJ, Stewart D, Pereira-Caro G, Gonzalez-Barrio R, Allsopp P, Magee P, Crozier A, Rowland I, Gill CI. (2012). PLoS One, 7, 1-10. 